\title{
Intractable Trigeminal Neuralgia Secondary to Osteoma of the Clivus: A Case Report and Literature Review
}

\author{
Ebtesam Abdulla ${ }^{1}$ Krishna Das ${ }^{2}$ Joseph Ravindra ${ }^{1}$ \\ ${ }^{1}$ Department of Neurosurgery, Salmaniya Medical Complex, \\ Manama, Bahrain \\ 2 Department of Neurosurgery, Lisie Hospital, Kerala, India \\ ${ }^{3}$ Department of Radiology, Salmaniya Medical Complex, Manama, \\ Bahrain \\ ${ }^{4}$ Department of Anatomical Pathology, Salmaniya Medical Complex, \\ Manama, Bahrain
}

J Neurosci Rural Pract 2022;13:141-145.

\author{
Tejal Shah ${ }^{3}$ Sara George ${ }^{4}$
}

\begin{abstract}
Keywords

- clivus

- osteoma

- skull base

Skull base osteomas (SBOs) are benign tumors that are frequently detected on radiographic images by coincidence. They are known for being slow-growing tumors and rarely symptomatic. The therapeutic approach for SBOs can differ substantially. Depending on the symptoms, size, and location of the tumor, this can range from serial observation to vigorous surgical extirpation. Clival osteoma is extremely rare. We report a case of clival osteoma, causing intractable trigeminal neuralgia due to the pressure effect on the trigeminal nerve at Meckel's cave. We also provide a review of pertinent literature. A 37-year-old woman presented with intractable trigeminal neuralgia. Cranial magnetic resonance imaging (MRI) demonstrated a large, lobulated, extra-axial lesion involving the right cerebellopontine angle and epicentering the clivus. Pathologically, the specimen was proven to be osteoma. The patient reported complete symptom resolution over a 4-year follow-up period. To the best of the authors' knowledge, this is the first clinical case of intractable trigeminal neuralgia due to clival osteoma.
\end{abstract}

\author{
Address for correspondence Ebtesam Abdulla, B.Med.Sc., MD, \\ Neurosurgery Resident, Department of Neurosurgery, Salmaniya \\ Medical Complex, Manama, Bahrain \\ (e-mail: Dr.Ebtesam@hotmail.com).
}

\section{Introduction}

Osteomas are made up of dense, mature bone, and exhibit benign features. ${ }^{1-19}$ They mostly arise from the calvarium and the mandible. ${ }^{1-4}$ Skull base osteomas (SBOs) are relatively rare; however, they are considered the most commonly benign, paranasal sinus tumors with a point incidence of 0.4 to $1 \%$ of the general population. ${ }^{1,2,5-11}$

published online January 7, 2022
DOI https://doi.org/

10.1055/s-0041-1742118. ISSN 0976-3147.
Osteomas are more common in males, with a male-tofemale ratio of 1.5 to $3: 1$. $^{1,2,4,6-10,13}$ SBO is most commonly found involving the anterior skull base in or around the sinonasal cavities. ${ }^{1-20}$ However, osteoma may arise from the middle and posterior skull base. ${ }^{11,14,16,18,20}$ Clival osteoma is extremely rare and has not been reported yet. Here, we report a clinical case of intractable trigeminal neuralgia due to clival osteoma, with a relevant literature review. (c) 2022. Association for Helping Neurosurgical Sick People. All rights reserved.

This is an open access article published by Thieme under the terms of the Creative Commons Attribution-NonDerivative-NonCommercial-License, permitting copying and reproduction so long as the original work is given appropriate credit. Contents may not be used for commercial purposes, or adapted, remixed, transformed or built upon. (https://creativecommons.org/ licenses/by-nc-nd/4.0/)

Thieme Medical and Scientific Publishers Pvt. Ltd., A-12, 2nd Floor, Sector 2, Noida-201301 UP, India 


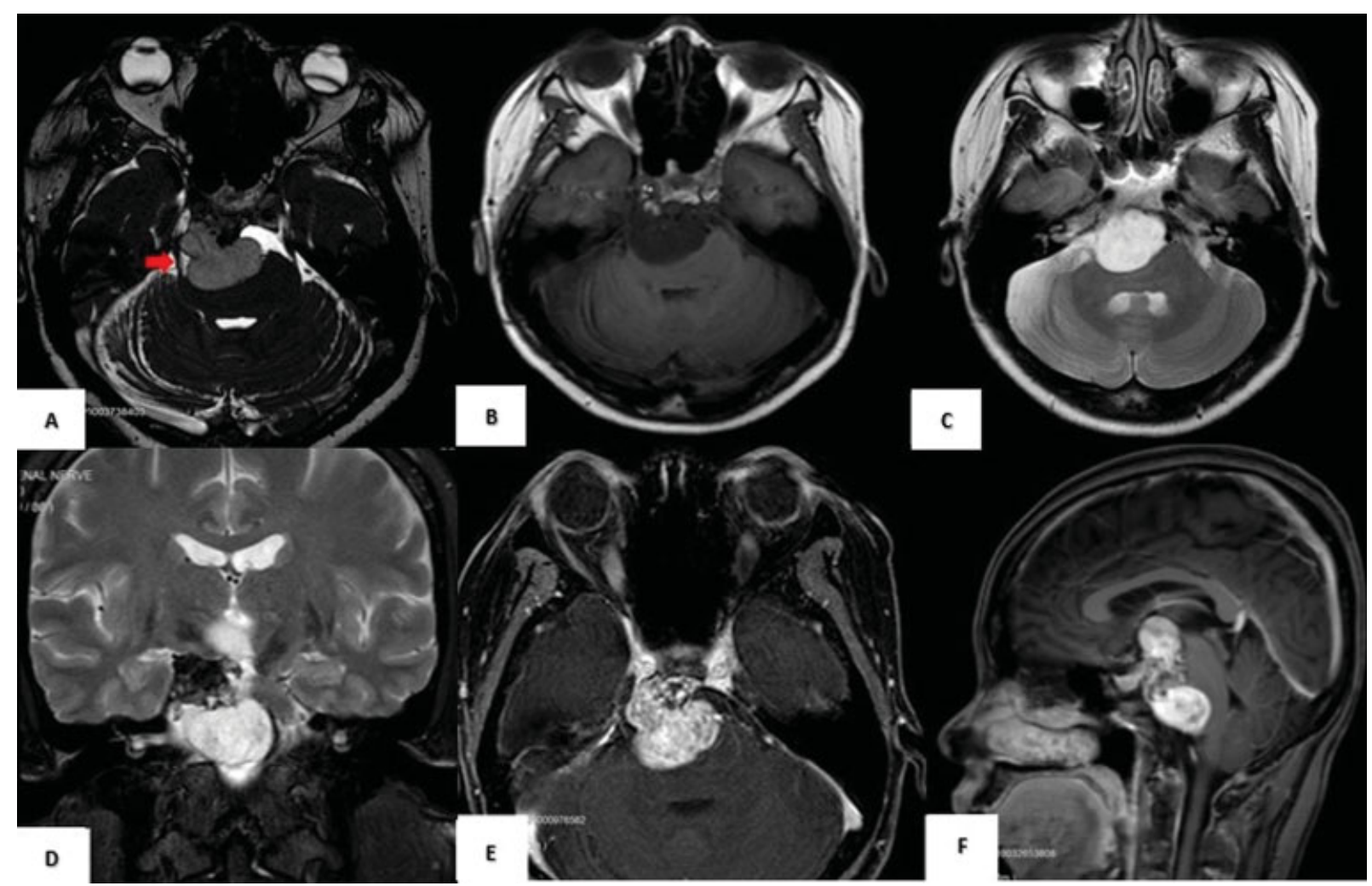

Fig. 1 Preoperative brain MRI shows a mildly compressing mass on the right trigeminal nerve at its cisternal part and the level of Meckel's cave (red arrow) Constructive Interference Steady State sequence (A). The mass is hypointense on T1-W (B), hyperintense on T2-W (C), not suppressed on Short Tau Inversion Recovery sequence (D), and vividly enhanced after contrast (E and F). The mass extends from the third ventricle's anterior aspect superiorly to the pontomedullary junction inferiorly and compresses the midbrain and pons along the right anterolateral aspect (F). MRI, magnetic resonance imaging; W, weighted.

\section{Case Description}

A 37-year-old woman endorsed a 3-year history of severe, lancinating, paroxysmal pain involving the right lower half of the face extending to the right lower half of the teeth, gums, and lip. The pain was provoked by speech and mastication. She initially had relief with oxcarbazepine and pregabalin but later became refractory to drugs. Her neurological evaluation was normal, aside from hyperesthesia in the right V3 division. A dental check-up was normal.

Cranial magnetic resonance imaging (MRI) demonstrated a large, lobulated, and extra-axial lesion in the area of the right cerebellopontine angle region which epicenter the clivus and caused compression of the right trigeminal nerve fibers at the level of Meckel's cave (-Fig. 1A). The mass displayed hypointense signal intensity on T1-weighted images ( - Fig. 1B), predominantly hyperintense signal intensity on $\mathrm{T} 2$-weighted images ( - Fig. 1C), and vivid enhancement in the postcontrast T1-weighted images (-Fig. 1E and $\mathbf{F}$ ). The mass was not displaying suppression in the T2-weighted fat saturation images (- Fig. 1D). No other abnormalities were seen on MRI. The differential diagnosis entertained was exophytic lesion arising from the clivus, schwannoma, and less likely meningioma.

The patient underwent right retrosigmoid craniectomy and decompression of retroclival mass. The mass was hard and calcified with soft elements in between arising from the clivus. The trigeminal nerve was seen displaced and compressed by the mass ( - Fig. 2A). We mainly aimed to decompress the trigeminal nerve and relieve the facial pain. It was

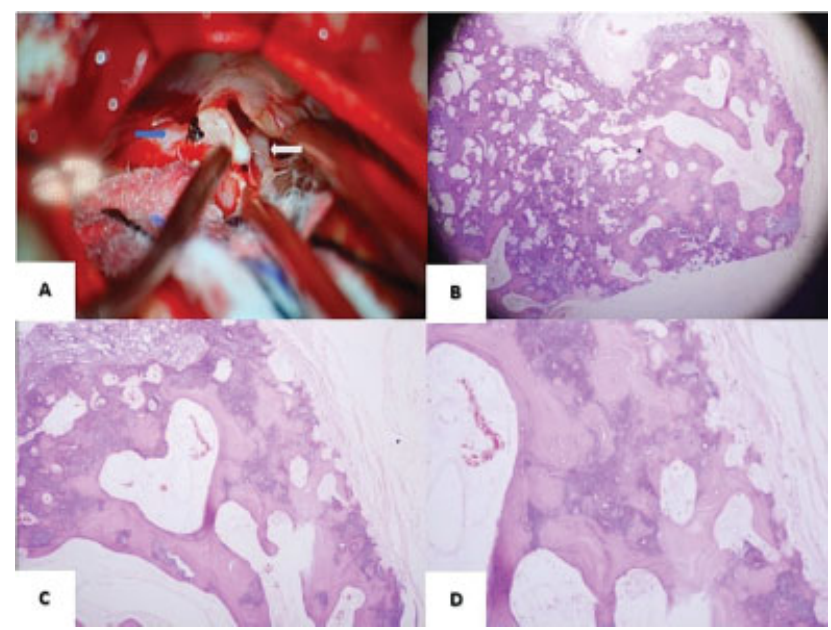

Fig. 2 An intraoperative photograph showing osteoma that arises from the clivus (blue arrow) and compresses the trigeminal nerve (white arrow) (A). Histopathology of the clival mass shows osteoma with a capsule, $H \& E$ stain, $\times 40$ magnification (B). The low power view shows the osteoma composed of trabeculae of mature bone enclosing empty marrow spaces with capsular tissue in the periphery, H\&E stain, $\times 100$ magnification $(C)$. The intermediate power view shows the trabeculae of mature bone with the lacunae containing the osteoblast, H\&E stain, $\times 200$ magnification (D). H\&E, hematoxylin and eosin. 


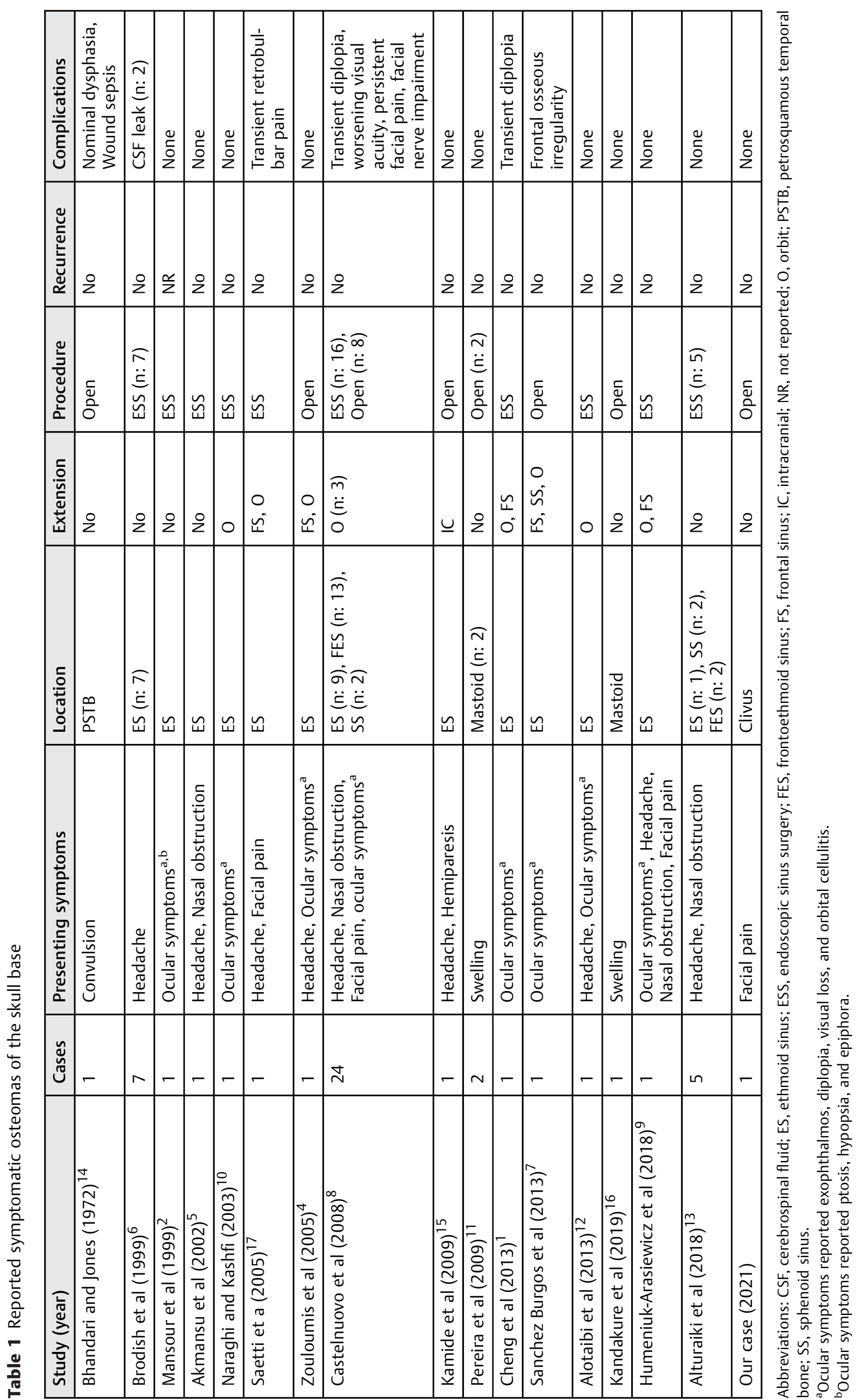


difficult to tackle the whole tumor due to its diffuse nature and vascularity. Thus, total extirpation was not attempted. A diagnosis of Clival osteoma was obtained by histopathology (-Fig. 2B-D). The resected mass revealed trabeculae of mature lamellated bone enclosing marrow spaces. No hemopoietic or malignant cells or cartilaginous tissue were detected. The patient recovered well postoperatively. She was discharged on oxcarbazepine and reported a complete symptom resolution over a 4-year follow-up period.

\section{Discussion}

SBOs are rare, nonmalignant tumors of uncertain origin. There are three etiologies postulated for osteomas. One is genetic while the others are traumatic causing muscle traction, and inflammatory ${ }^{2,3,20}$; however, the exact etiology for osteomas' occurrence is unclear.

We conducted a literature search of PubMed and Google Scholar databases on June 20, 2021. We found 50 reported cases of SBOs (-Table 1). Adding our case, SBOs were predominately seen in adults with a male predilection. The vast majority of SBOs were unilateral (98\%), and only one case was bilateral $(1.9 \%)^{2}$ Presenting symptoms (-Table 2 ) mainly were mass effect-type symptoms depending on their location and including headache (62.7\%), nasal obstruction (31.3\%), ocular symptoms (15.6\%), facial pain (7.85\%), and

Table 2 Clinical characteristic of all reported and available skull base osteomas

\begin{tabular}{|l|l|l|}
\hline Skull base osteomas & $n$ & $\%$ \\
\hline Total number & 51 & 100 \\
\hline Initial symptoms & & \\
\hline Headache & 32 & 62.7 \\
\hline Nasal obstruction & 16 & 31.3 \\
\hline Ocular symptoms & 8 & 15.6 \\
\hline Facial pain & 4 & 7.84 \\
\hline Swelling & 3 & 5.88 \\
\hline Hemiparesis & 1 & 1.96 \\
\hline Convulsion & 1 & 1.96 \\
\hline Origin & & \\
\hline Ethmoid sinus & 27 & 52.9 \\
\hline Frontoethmoidal sinus & 15 & 29.4 \\
\hline Sphenoid sinus & 4 & 7.84 \\
\hline Mastoid & 3 & 5.88 \\
\hline PSTB & 1 & 1.96 \\
\hline Clivus & 1 & 1.96 \\
\hline Treatment & & \\
\hline Open surgery & 16 & 31.3 \\
\hline Endoscopic sinus surgery & 35 & 68.6 \\
\hline
\end{tabular}

Abbreviation: PSTB, petrosquamous temporal bone. apparent mass (5.88\%). On the other hand, other rare presenting symptoms have also been reported, such as hemiparesis (1.96\%) and convulsions (1.96\%), seemingly due to intracranial tumor extension. Our patient had a history of intractable trigeminal neuralgia which manifested as facial pain. We have seen that osteoma originates from the clivus and extends into the cerebellopontine angle. Therefore, we presumed that the patient's facial pain was primarily due to the osteoma's mass effect, pushing and compressing the trigeminal nerve fiber at the Meckel's cave.

A cranial computed tomography (CT) scan was required for radiological diagnosis ( - Table 2 ). A CT scan demonstrated features consistent with low-grade bony tumors. They identified a juxtacortical, well-circumscribed sclerotic lesion with the preservation of diploe that did not infiltrate the surrounding soft tissues. On MRI, osteomas are hypointense on T1-weighted images with variable signal intensities on T2-weighted images based on the amount of cancellous bone and compact bone. ${ }^{19}$ The bulk of the tumors were confined to the ethmoid sinus (27 cases), frontoethmoidal sinus (15 cases), sphenoid sinus (4 cases), mastoid ( 3 cases), petrosquamous temporal bone ( 1 case), and clivus ( 1 case). There was tumor extension to the orbit (10 cases) and frontal sinus (5 cases), mainly from the ethmoid-based osteomas. Sphenoid sinus (1 case) and intracranial (1 case) extensions have been reported. The size of the osteomas varied with the maximum reported $5 \mathrm{~cm}$. In our case, we performed a cranial MRI to evaluate for trigeminal neuralgia. However, we could not do a CT scan to confirm the diagnosis for technical reasons, and the option for surgery was decided.

The differential diagnosis of SBOs should consider the following: osteoid osteoma, ossifying hemangioma, ossifying fibroma, calcified meningioma, and malignant lesions. ${ }^{2,7,11,16,19}$ In the present case, a sufficient sample was sent for pathological analysis which was typical for compact bone. No hematopoietic or malignant cells or cartilaginous tissues were detected.

In the present view, 35 patients of 51 symptomatic cases underwent endoscopic sinus surgery (ESS), and only 16 patients underwent open surgery. ESS or open surgery's decision mainly depends on the site and the tumor's size and the surgeon's preference. ${ }^{1-18}$ In our case, the aim was to decompress the trigeminal nerve. Hence, open surgery was decided on through a retrosigmoid approach. In the literature, most surgeons attempted total osteoma extirpation and reported no tumor recurrence. However, total osteoma extirpation is not always achievable. In our case, a total extirpation of clival osteoma was not achieved due to its total diffuse nature and vascularity. SBO surgery is relatively safe. However, some patients reported postoperative complications such as nominal dysphasia, wound sepsis, cerebrospinal fluid leak, transient retrobulbar pain, symptoms persistence or worsening, facial nerve impairment, and facial osseous irregularity. Although SBOs seldom recur after surgery, Zouloumis et al and Brodish et al described recurrent cases successfully managed by total extirpation. $^{4,6}$ 


\section{Conclusion}

Osteoma originating from the clivus is extremely rare; however, it should be included as a differential diagnosis of intractable trigeminal neuralgia. The histopathological assessment is the gold-standard test for diagnosis. Furthermore, microsurgical decompression for trigeminal neuralgia has a good neurological outcome.

\section{Authors' Contributions}

E.A.: conceptualization, methodology, -preparing the original draft, review and editing, and formal analysis; K.D.: data curation and visualization; J.R.: conceptualization, data curation, visualization, review, and supervision; T.S.: preparing manuscript, review and editing, and supervision; And S.G.: data collection and preparing manuscript.

Note

This article does not record previous presentation in a scientific meeting.

Funding

None.

\section{Conflict of Interest}

None declared.

\section{References}

1 Cheng KJ, Wang SQ Lin L. Giant osteomas of the ethmoid and frontal sinuses: Clinical characteristics and review of the literature. Oncol Lett 2013;5(05):1724-1730

2 Mansour AM, Salti H, Uwaydat S, Dakroub R, Bashshour Z. Ethmoid sinus osteoma presenting as epiphora and orbital cellulitis: case report and literature review. Surv Ophthalmol 1999;43 (05):413-426

3 Kaplan I, Calderon S, Buchner A. Peripheral osteoma of the mandible: a study of 10 new cases and analysis of the literature. J Oral Maxillofac Surg 1994;52(05):467-470

4 Zouloumis L, Lazaridis N, Maria P, Epivatianos A. Osteoma of the ethmoidal sinus: a rare case of recurrence. $\mathrm{Br} \mathrm{J}$ Oral Maxillofac Surg 2005;43(06):520-522
5 Akmansu H, Eryilmaz A, Dagli M, Korkmaz H. Endoscopic removal of paranasal sinus osteoma: a case report. J Oral Maxillofac Surg 2002;60(02):230-232

6 Brodish BN, Morgan CE, Sillers MJ. Endoscopic resection of fibroosseous lesions of the paranasal sinuses. Am J Rhinol 1999;13 (02):111-116

7 Sanchez Burgos R, González Martín-Moro J, Arias Gallo J, Carceller Benito F, Burgueño García M. Giant osteoma of the ethmoid sinus with orbital extension: craniofacial approach and orbital reconstruction. Acta Otorhinolaryngol Ital 2013;33(06):431-434

8 Castelnuovo P, Valentini V, Giovannetti F, Bignami M, Cassoni A, Iannetti G. Osteomas of the maxillofacial district: endoscopic surgery versus open surgery. J Craniofac Surg 2008;19(06): 1446-1452

9 Humeniuk-Arasiewicz M, Stryjewska-Makuch G, Janik MA, Kolebacz B. Giant fronto-ethmoidal osteoma - selection of an optimal surgical procedure. Rev Bras Otorrinolaringol (Engl Ed) 2018;84 (02):232-239

10 Naraghi M, Kashfi A. Endonasal endoscopic resection of ethmoido-orbital osteoma compressing the optic nerve. Am J Otolaryngol 2003;24(06):408-412

11 Pereira CU, Carvalho RWF, Almeida AMG, et al. Mastoid osteoma. consideration on two cases and literature review. Int Arch Otorhinolaryngol 2009;13(03):346-349

12 Alotaibi N, Hanss J, Benoudiba F, Bobin S, Racy E. Endoscopic removal of large orbito-ethmoidal osteoma in pediatric patient: case report. Int J Surg Case Rep 2013;4(12):1067-1070

13 Alturaiki SM, Almomen A, Azzeh GA, et al. Fibro-osseous lesions of the paranasal sinuses and the skull base. Int J Otorhinolaryngol Head Neck Surg 2018;4:1324-1330

14 Bhandari YS, Jones RA. Osteoma of the middle cranial fossa. Case report. J Neurosurg 1972;37(05):610-612

15 Kamide T, Nakada M, Hayashi Y, Hayashi Y, Uchiyama N, Hamada J. Intraparenchymal pneumocephalus caused by ethmoid sinus osteoma. J Clin Neurosci 2009;16(11):1487-1489

16 Kandakure VT, Lahane VJ, Mishra S. Osteoma of mastoid bone; a rare presentation: case report. Indian J Otolaryngol Head Neck Surg 2019;71(Suppl 2):1030-1032

17 Saetti R, Silvestrini M, Narne S. Ethmoid osteoma with frontal and orbital extension: endoscopic removal and reconstruction. Acta Otolaryngol 2005;125(10):1122-1125

18 Shimizu T, Okamoto K, Majima Y. Osteoma of the malleus: a case report and literature review. Am J Otolaryngol 2003;24(04):239-241

19 Lloret I, Server A, Taksdal I. Calvarial lesions: a radiological approach to diagnosis. Acta Radiol 2009;50(05):531-542

20 Thomas R. Familial osteoma of the middle ear. J Laryngol Otol 1964;78:805-807 